\title{
Perfil hormonal, metabólico y hematológico en adultos con el Virus de Inmunodeficiencia Humana
}

\author{
Hormonal, metabolic and hematological profile in \\ adults with the Human Immunodeficiency Virus
}

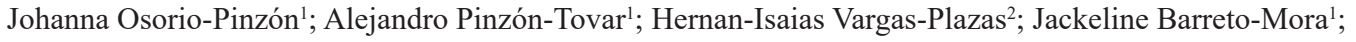 \\ Deiner-Yivelson Muñoz-Delgado 3 ; Diego Santana-Velasco ${ }^{3}$; Carlos Vanegas- Vanegas ${ }^{3}$; Johana Imbachí-Ramos ${ }^{3}$
}

Forma de citar: Osorio-Pinzón J, Pinzón-Tovar A, Vargas-Plazas HI, Barreto-Mora J, Muñoz-Delgado DY, Santana-Velasco D, et al. Perfil hormonal, metabólico y hematológico en adultos con el Virus de Inmunodeficiencia Humana. Rev Univ Ind Santander Salud. 2018; 50(4): 296-306. doi: http://dx.doi.org/10.18273/revsal.v50n4-2018003 @c) (i)

\section{RESUMEN}

Introducción: Son escasos los datos del impacto de la infección por el virus de inmunodeficiencia humana (VIH) a nivel hormonal, metabólico y hematológico en pacientes hospitalizados en Colombia. Objetivo: Describir el perfil hormonal, metabólico y hematológico de los pacientes con VIH hospitalizados en una institución de tercer nivel. Material y método: Estudio observacional de corte transversal, donde se incluyeron variables sociodemográficas, clínicas, hormonales, metabólicas y hematológicas de pacientes con VIH entre el 2013 y 2014. Resultados: Se incluyeron 52 pacientes, 34 hombres, con media de edad 39,7 años (Ds 12,6, Min: 21 Max: 79). 23\% habían cursado con tuberculosis, $13 \%$ con toxoplasmosis cerebral. 26 pacientes tenían historia de consumo de tóxicos: cigarrillo (58\%), alcohol (27\%) y sustancias alucinógenas (15\%). 14\% de los pacientes recibían terapia antirretroviral al ingreso, los esquemas contenían principalmente inhibidores de proteasa. Otros medicamentos usados fueron: trimetoprim-sulfametoxazol $(27.2 \%)$ y antituberculosos (15\%). Las principales causas de hospitalización fueron toxoplasmosis cerebral (31\%) y tuberculosis $(15 \%) .52 \%$ de la población presentó síndrome de desgaste. El tiempo de diagnóstico del VIH fue $<1$ de un año en el $48 \%$ de la población. $79 \%$ de los pacientes tenía recuento de CD4 <200cel $/ \mathrm{mm}^{3}$ (Ds 199, Min: 3 Max: 641). En el perfil hormonal, 58\% (29 pacientes) presentaron alteración del eje tiroideo, de los cuales 14 presentaron perfil de hipotiroidismo central. 55,8\% de los hombres presentaron hiperprolactinemia. El perfil metabólico se caracterizó por hipertrigliceridemia (44\%) y HDL bajas (81\%). La alteración electrolítica de mayor frecuencia fue hiponatremia (37\%). Conclusiones: En la población de pacientes hospitalizados con VIH, se encontraron alteraciones endocrinas que sugieren compromiso glandular primario hipofisiario y adrenal; alteraciones lipídicas y electrolíticas en gran medida relacionadas con enfermedad avanzada.

Palabras Clave: Virus de la Inmunodeficiencia Humana; Hormonas; Metabólico; Electrolitos; Hospitalizacion; Colombia.

\footnotetext{
1. Hospital Universitario de Neiva. Huila, Colombia

2. Clínica Medilaser - Neiva. Huila, Colombia

3. Universidad Surcolombiana - Neiva. Huila, Colombia

Correspondencia: Deiner Yivelson Muñoz Delgado. Dirección: Calle 18B 33A-37 Neiva-Huila, Colombia. Teléfono: +57 3112522571 . Correo electrónico: deineryiv@hotmail.com.
} 


\begin{abstract}
Introduction: Data on the impact of human immunodeficiency virus (HIV) infection on a hormonal, metabolic and haematological level in patients hospitalized in Colombia are scarce. Objective: To describe the hormonal, metabolic and haematological profile of HIV patients hospitalized in a third level institution. Material and method: Crosssectional observational study, which included sociodemographic, clinical, hormonal, metabolic and hematological variables of patients with HIV between 2013 and 2014. Results: We included 52 patients, 34 men, with an average age of 40 years (SD 12.6, Min: 21 Max: 79). 23\% had tuberculosis, 13\% had cerebral toxoplasmosis. 26 patients had a history of toxic consumption: cigarette (58\%), alcohol (27\%) and hallucinogenic substances (15\%). 14\% of the patients received antiretroviral therapy at admission, mainly with protease inhibitors. Other medications used were: trimethoprim-sulfamethoxazole (27.2\%) and antituberculous drugs (15\%). The main causes of hospitalization were cerebral toxoplasmosis $(31 \%)$ and tuberculosis $(15 \%) .52 \%$ of the population had Wasting syndrome. The time of diagnosis of HIV was $<1$ year in $48 \%$ of the population. $79 \%$ of the patients had a CD4 count $<200$ cell $/ \mathrm{mm} 3$ (SD 199, Min: 3 Max: 641). In the hormonal profile, 58\% (29 patients) presented alteration of the thyroid axis, of which 14 session profile of central hypothyroidism. 55.8\% of men had hyperprolactinemia. The metabolic profile was characterized by hypertriglyceridemia (44\%) and low HDL (81\%). The most frequent electrolyte alteration was hyponatremia (37\%). Conclusions: In the population of patients hospitalized with HIV, endocrine alterations were found suggesting primary glandular, pituitary and adrenal involvement, with lipid and electrolyte alterations largely related to advanced disease.
\end{abstract}

Keywords: Human Immunodeficiency Virus; Hormones; Metabolic; Hospitalization; Colombia.

\section{Introducción}

La infección por el Virus de Inmunodeficiencia Humana (VIH) continúa en aumento. Para junio del año 2015, aproximadamente 36,7 millones de personas en todo el mundo estaban infectadas según el Programa Conjunto de las Naciones Unidas sobre el VIH/SIDA (ONUSIDA); para este mismo año 2,1 millones de personas contrajeron la infección por VIH en el mundo y 1,1 millones de personas fallecieron por causas relacionadas con el SIDA ${ }^{1}$. En Colombia, de acuerdo a la información epidemiológica de 2016, se notificaron 13.005 casos nuevos confirmados por laboratorio ${ }^{2}$. En el Huila hasta la semana epidemiológica 32 del 2016 se notificaron 95 casos nuevos de VIH/SIDA y desafortunadamente, entre un $12 \%$ a $14 \%$ de los pacientes con VIH que son hospitalizados, fallecen ${ }^{3}$.

El desarrollo de la terapia antirretroviral altamente efectiva (Highly Active Antiretroviral Therapy, HAART) ha trasformado el curso de la infección por $\mathrm{VIH}$, en su incidencia, el manejo de las infecciones oportunistas (IO) y descenso en la mortalidad asociada al $\mathrm{VIH}^{29}$.

El VIH afecta principalmente el sistema inmunitario, exponiendo a la persona a infecciones por microorganismos oportunistas, sin embargo, se han descrito alteraciones en otros sistemas de tipo hematológico, endocrinológico y metabólico, entre $\operatorname{otros}^{3,4}$.
Desde el año 1992, se ha descrito la relación del VIH con el sistema endocrino siendo los cambios más importantes la insuficiencia suprarrenal y las alteraciones en el eje tiroideo. También se han reportado alteraciones del eje renina - angiotensina aldosterona, resistencia a la insulina, diabetes mellitus, trastornos del metabolismo óseo, alteración del eje gonadal y trastornos metabólicos (hipertrigliceridemia, hipercolesterolemia y niveles bajos de lipoproteínas de alta densidad (HDL) ${ }^{4}$. Las alteraciones endocrinas en pacientes con VIH son multifactoriales e incluyen mediadores de respuesta inflamatoria sistémica, pobre estado nutricional, infecciones oportunistas, efectos directos del VIH, destrucción del tejido endocrino, interferencia con la función endocrina, entre otras ${ }^{4}$. Los signos y síntomas predominantes en estos pacientes son: fiebre, síntomas constitucionales (astenia, adinamia, anorexia, pérdida de peso), respiratorios, cefalea, trastornos gastrointestinales, entre otros ${ }^{3}$. Las alteraciones endocrinas y metabólicas pueden ocurrir como una complicación de la terapia antirretroviral o debido al efecto directo de la infección viral ${ }^{5}$.

En nuestro medio no hay documentación amplia que describa los trastornos endocrinos, metabólicos y hematológicos de los pacientes con VIH hospitalizados. Es por ello que nuestro trabajo pretende aportar datos a la epidemiologia nacional y regional, especialmente de la población que requiere hospitalización por infecciones oportunistas $u$ otras condiciones asociadas al VIH. 
El objetivo de este estudio fue describir el perfil hormonal y metabólico de los pacientes con VIH hospitalizados en una institución de tercer nivel de Colombia.

\section{Materiales y métodos}

\section{Tipo de estudio}

Se realizó un estudio observacional, de corte transversal, en pacientes con diagnostico VIH/SIDA hospitalizados en el Hospital Universitario Hernando Moncaleano Perdomo (HUHMP), Neiva, Huila, Colombia. El periodo de reclutamiento fue de 2013 a 2014.

\section{Sitio de estudio}

Hospital Universitario Hernando Moncaleano Perdomo de Neiva, es una institución de tercer nivel de complejidad, centro de referencia del sur colombiano, cuenta con una unidad especial en el servicio de hospitalización de infectologia adultos, con siete camas; la mayor población atendida pertenece al régimen subsidiado.

\section{Criterios de inclusión}

Se incluyeron todos los pacientes mayores de 18 años con diagnóstico confirmado de infección por VIH hospitalizados en el HUHMP en el periodo comprendido entre el 1 de septiembre de 2013 hasta el 1 de septiembre de 2014; 52 pacientes cumplieron con los criterios de selección.

\section{Criterios de exclusión}

Se excluyeron pacientes que fallecieron durante la hospitalización antes de la toma de paraclínicos y aquellos con reingreso hospitalario en el periodo de estudio.

Las pruebas se realizaron en el Laboratorio Clínico del hospital Universitario de Neiva. Antes de la toma de las muestras, todos los sujetos dieron su consentimiento informado.

\section{Medición y definición operativa de las variables}

Se incluyeron variables sociodemográficas, clínicas y paraclínicas. En cuanto las variables paraclínicas se tuvieron en cuenta parámetros hormonales, metabólicos y hematológicos.
Para la recolección de la información de los datos sociodemográficos y clínicos, los autores utilizaron un instrumento de recolección de datos previamente validado en una prueba piloto por los mismos con base en la información de la historia clínica y en aquella recolectada a través de entrevista directa realizada a los pacientes, previa explicación y firma de consentimiento informado.

Se tuvieron en cuenta varios mecanismos de control de calidad del dato, de los cuales se puede mencionar la preparación del instrumento de recolección de datos, prueba piloto, desarrollo de procedimientos para la recolección y procesamiento de datos con entrenamiento a los autores para la realización de dicho proceso y corrección de los errores descubiertos durante el mismo.

La información recolectada se almacenó en una base de datos en Excel y posteriormente se analizaron las variables cualitativas y cuantitativas en el programa estadístico Epi Info 7.

Para las variables hormonales, se obtuvieron muestras en sangre al ingreso para la medición de la hormona estimulante del tiroides (TSH), tiroxina T4 libre, hormona folículo estimulante (FSH), hormona luteinizante (LH), Somatomedina c, Hormona de crecimiento, Vitamina D, estradiol y prolactina. El cortisol fue tomado en las horas de la mañana tanto para hombres como mujeres y la testosterona libre fue tomada solo en hombres.

Para las variables metabólicas, se obtuvieron muestras de sangre al ingreso del paciente para medición de glicemia basal, colesterol total, lipoproteínas de baja densidad (LDL), lipoproteínas de alta densidad (HDL), lipoproteínas de muy baja densidad (VLDL), triglicéridos, albúmina y proteínas totales. Se consideró como hipercolesterolemia aquel valor de colesterol total mayor o igual a $200 \mathrm{mg} / \mathrm{dl}$ y como hipertrigliceridemia valores mayores o iguales a 150 $\mathrm{mg} / \mathrm{dl}$. Los niveles de HDL por debajo de $35 \mathrm{mg} / \mathrm{dl}$, se consideraron como bajos y los niveles de LDL mayores o iguales a $130 \mathrm{mg} / \mathrm{dl}$ se consideraron altos. Hiperglucemia se definió como aquel valor superior o igual a $100 \mathrm{mg} / \mathrm{dl}$ en ayunas.

En la química sanguínea se determinó nitrógeno ureico (BUN), creatinina, transaminasa glutámico oxalacética (TGO), transaminasa glutámico pirúvica (TGP) y electrolitos como sodio, potasio y calcio iónico. 
Para la medición de las variables hematológicas se realizó hemograma completo y recuento de linfocitos T-CD4. A cada paciente al momento de ingresar al hospital, se le realizó un hemograma automatizado. Se definió como anemia la hemoglobina menor de $12 \mathrm{~g} /$ $\mathrm{dL}$ en la mujer y de $13 \mathrm{~g} / \mathrm{dL}$ en el hombre, leucopenia como un conteo de leucocitos menor que $4 \times 10^{9} / \mathrm{L}$ y trombocitopenia como un conteo de plaquetas menor a $150 \times 10^{9} /$ L. El Volumen Corpuscular Medio (VCM) se definió como normal entre 80 y 100 fL. Se consideró pancitopenia la alteración cuantitativa de las tres líneas celulares (anemia, leucopenia y trombocitopenia) y se definió como bicitopenia la alteración de dos líneas celulares.

Finalmente, durante la hospitalización a cada paciente se le realizó determinación del estado inmunológico mediante la cuantificación de linfocitos T-CD4.

\section{Análisis estadístico}

El análisis estadístico fue descriptivo de acuerdo a la distribución de las variables. Las variables cuantitativas se analizaron mediante la obtención de la media con su desviación estándar (Ds) o medianas con rango intercuartílico (RIQ). Las variables cualitativas se expresaron con frecuencias y proporciones.

\section{Limitaciones del estudio}

No fue posible realizar la medición de vitamina D y somatomedina $\mathrm{C}$; por otro lado, algunas pruebas no fueron reportadas en la mayoría de pacientes, como ocurrió, con el estradiol (8/52) y la testosterona (10/52).

Otra limitación fue el pequeño tamaño de la muestra. Tampoco se contó con un grupo de control de pacientes hospitalizados sin condiciones definitorias de SIDA o pacientes con VIH en categoría A o B del CDC.

Un aspecto importante por mencionar, fue el excluir a pacientes hospitalizados con VIH que fallecieron antes de terminar de tomar todas las muestras sanguíneas, ya que ello pudo conllevar al desconocimiento del estado clínico y paraclínico de los pacientes más graves.

\section{Resultados}

\section{Caracterizacion sociodemográfica}

En el HUHMP se reclutaron 52 pacientes durante 12 meses, que cumplieron los criterios de inclusión y exclusión. El 65\% eran hombres (34/52). El 42\% del total de la población se encontraba en edad reproductiva, con una media de edad 39,7 (Ds 12,6 ;Min: 21-Max 79 años). $54 \%$ eran solteros, $54 \%$ escolaridad primaria y el $98 \%$ pertenecían a los estratos socioeconómicos 1 y 2 (Tabla 1).

Tabla 1. Características sociodemográficas de pacientes con infecciòn por VIH hospitalizados en el HUHMP.

\begin{tabular}{lc}
\hline \multicolumn{1}{c}{ Variable } & $\boldsymbol{n}=\mathbf{5 2}(\boldsymbol{\%})$ \\
\hline Edad: & $\mathbf{3 9 . 7 ( 1 2 . 6 ) *}$ \\
$\mathbf{1 8}-35$ años & $22(42.3)$ \\
$36-60$ años & $26(50.0)$ \\
$>60$ años & $4(7.6)$ \\
Género & \\
Masculino & $34(65.3)$ \\
Femenino & $18(34.6)$ \\
Escolaridad & \\
Universidad & $1(1.9)$ \\
Ninguno & $4(7.6)$ \\
Secundaria & $19(36.5)$ \\
Primaria & $28(53.8)$ \\
Estado civil & \\
Viudez & $2(3.8)$ \\
Casado & $9(17.3)$ \\
Unión Libre & $13(25.0)$ \\
Soltero & $28(53.8)$ \\
Estrato & \\
1 & $43(82.6)$ \\
3 & $8(15.3)$ \\
4 & $0(0.0)$ \\
\hline
\end{tabular}

Frecuencia ( $\mathrm{n}=52)$, Porcentaje (\%), HUHMP: Hospital Universitario Hernando Moncaleano Perdomo.

* para esta variable en esta celda se presenta la media y la desviación estándar.

\section{Características clínicas}

En el 48\% (25/52) de los participantes el diagnóstico de infección por VIH era menor de un año, con mediana de 1 año, con rango mínimo y máximo de tiempo de diagnóstico menor de 1 a 13 años y rango intercuartilico de 3 años (Figura 1). Como antecedentes patológicos, dos pacientes cursaban con hipertensión arterial, solo uno recibía tratamiento antihipertensivo, uno tenía hipotiroidismo en suplencia hormonal con levotiroxina y otro tenía enfermedad renal crónica. Se reportaron enfermedades oportunistas en un 64\%, con mayor frecuencia tuberculosis $23 \%$ y toxoplasmosis cerebral $13 \%$. En cuanto a antecedentes quirúrgicos, 11 de los pacientes habían tenido alguna intervención quirúrgica 
siendo la cesárea la de mayor frecuencia; cinco pacientes presentaron antecedente de trauma craneal. 26 de los 52 pacientes consumían sustancias tóxicas, así: $58 \%$ cigarrillo, $27 \%$ alcohol y $15 \%$ sustancias alucinógenas.

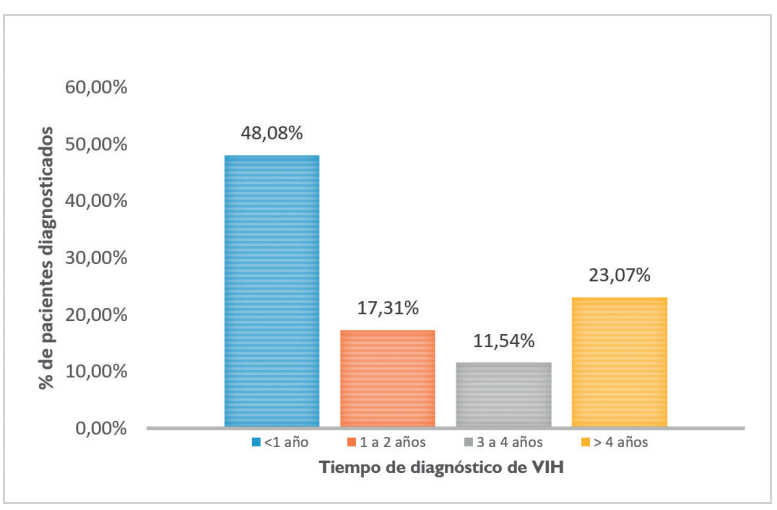

Figura 1. Tiempo de diagnóstico de los pacientes con infección por VIH hospitalizados en el HUHMP.

Las principales causas de hospitalización fueron toxoplasmosis cerebral $(31 \% ; 16 / 52)$, tuberculosis $(15 \% ; 8 / 52)$ y candidiasis $(15 \% ; 8 / 52)$. El $42,3 \%$ (22/52 de los pacientes estudiados se encontraban en bajo peso y $57,7 \%(30 / 52)$ en peso normal; ningún paciente presentó sobrepeso, estimado con el Índice de Masa Corporal (IMC), con media de 20, desviación estándar de 2 con rangos para hombres mínimo de 15,6 y máximo de 23,8 y para mujeres mínimo de 15,6 y máximo de 23,1 (Tabla 2). El 52\% de los pacientes presentaron síndrome de desgaste descrito como la pérdida involuntaria de al menos $10 \%$ del peso corporal inicial en presencia de diarrea, fiebre o debilidad crónica. Con respecto al tiempo de hospitalización se presentó una mediana de 32 días, rango intercuartílico (RIQ 1): 17 y (RIQ 3): 27.

En cuanto a la terapia antirretroviral combinada (ARTc), solo $14 \%(7 / 52)$ la recibían de forma regular al ingreso, principalmente con Inhibidores de Proteasa (IP), Con relación a otros fármacos, recibían trimetoprim sulfametoxazol (TMP/SMX) 27\% y antituberculosos 15\% (Tabla 3).

\section{Caracterización del perfil endocrino}

En el análisis del eje hipofisario - adrenal, se encontró cortisol con niveles por debajo de $4.3 \mathrm{ug} / \mathrm{dl}$, en 12 hombres (41.3\%) y 5 mujeres (31.2\%) (Tabla 4$)$.

$\mathrm{Al}$ analizar el eje hipofisario tiroideo se encontró 58\% (29 pacientes) con alteración del eje tiroideo, de los cuales 14 presentaron perfil de hipotiroidismo central,
10 hipotiroidismo subclínico, tres hipotiroidismo primario y dos hipertiroidismo subclínico (Figura 2). Uno de los pacientes tenía hipotiroidismo como antecedente de importancia. En tres casos la TSH se encontraba dentro de parámetros normales, pero no se reportaron los resultados de T4 libre; 20 pacientes fueron eutiroideos.

Tabla 2. Características clìnicas de pacientes con infección por VIH hospitalizados en el HUHMP.

\begin{tabular}{lc}
\hline Variable & $\boldsymbol{n}=\mathbf{5 2}(\boldsymbol{\%})$ \\
\hline Índice de masa corporal & $19.5(2.2)^{*}$ \\
Bajo peso & $22(42.3)$ \\
Normal & $30(57.6)$ \\
Sobrepeso y obesidad & $0(0.0)$
\end{tabular}

Antecedente de consumo de sustancias tóxicas $(n=26 / 52)$

Cigarrillo

Alcohol

Sustancias alucinógenas

Ninguna

$33(63.4) * *$

Antecedentes de enfermedades oportunistas asociadas al VIH

Toxoplasmosis cerebral

Citomegalovirus

Linfoma

Sífilis

Pneumocystis Pulmonar

Otros

Enfermedades oportunistas padecidas durante la hospitalización

Toxoplasmosis cerebral

Tuberculosis

Candidiasis

Sífilis

Criptococosis meningea

Pneumocystis

Citomegalovirus

Linfoma

Otros

Tiempo de hospitalización

*32.5 (26.3)

Frecuencia $(\mathrm{n}=52)$, Porcentaje $(\%)$.

HUHMP: Hospital Universitario Hernando Moncaleano Perdomo. *para esta variable en la celda se presenta la media y la desviación estándar, ** Se realizó con respecto a $\mathrm{n}=52$. 
Tabla 3. Tratamiento farmacológico de los pacientes con infección por VIH hospitalizados en el HUHMP.

\begin{tabular}{|c|c|}
\hline Variables & Frecuencia \\
\hline $\begin{array}{l}\text { Regímenes de TAR (ART) combinada } \\
\text { iniciado previo al ingreso hospitalario }\end{array}$ & $\mathrm{n}=52(\%)$ \\
\hline (Lamivudina/Zidovudina) + Efavirenz & $1(1.9)$ \\
\hline $\begin{array}{l}\text { (Lamivudina/Zidovudina) }+ \text { (Lopinavir / } \\
\text { Ritonavir) }\end{array}$ & $2(3.8)$ \\
\hline $\begin{array}{l}\text { (Lamivudina/Zidovudina) + (Atazanavir/ } \\
\text { Ritonavir) }\end{array}$ & $1(1.9)$ \\
\hline $\begin{array}{l}\text { (Emtricitabina/ Tenofovir) }+ \text { (Lopinavir / } \\
\text { Ritonavir) }\end{array}$ & $2(3.8)$ \\
\hline $\begin{array}{l}\text { (Emtricitabina/ Tenofovir) }+ \text { (Atazanavir/ } \\
\text { Ritonavir) }\end{array}$ & $1(1.9)$ \\
\hline Subtotal & $7(13.4)$ \\
\hline $\begin{array}{l}\text { Regímenes de TAR (ART) combinada } \\
\text { iniciado durante la hospitalización }\end{array}$ & $5(9.6)$ \\
\hline Regímenes suspendidos & $2(3.8)$ \\
\hline Otros regímenes suspendidos & $3(5.7)$ \\
\hline Ninguno & $35(67.3)$ \\
\hline $\begin{array}{l}\text { Medicamentos utilizados para otras } \\
\text { patologías durante la hospitalización }\end{array}$ & $n=43(\%)$ \\
\hline Trimetoprim /Sulfametoxazol & $13(27.2)$ \\
\hline Antibacterianos & $8(18.6)$ \\
\hline Fluconazol & $5(11.6)$ \\
\hline Claritromicina & $4(9.3)$ \\
\hline Clindamicina & $3(6.9)$ \\
\hline Metronidazol & $2(4.6)$ \\
\hline Cefepime & $2(4.6)$ \\
\hline Otros & $10(23.2)$ \\
\hline
\end{tabular}

ART: Antiretroviral Therapy, HUHMP: Hospital Universitario Hernando Moncaleano Perdomo

En la Tabla 4 se representa el perfil hormonal. Con respecto al análisis del eje hipofisario gonadal, del total de la muestra, solo a 10 hombres se le tomaron niveles séricos de testosterona libre y de estos, dos presentaron hipogonadismo.

Al total de las mujeres en edad fértil (15) se le fue tomaron niveles séricos de FSH y LH, a ocho de 18 se les midieron niveles de estradiol, de las cuales cuatro se encontraron en falla ovárica.

Se encontró que 19 de 34 hombres $(55,8 \%)$ y ocho de18 mujeres (44\%), presentaron hiperprolactinemia (Tabla 4).

\section{Caracterización del perfil metabólico y} componentes de la química sanguínea

Los triglicéridos y VLDL se encontraron aumentados en $44 \%$ de los pacientes, $81 \%$ presentaron HDL baja y en cuanto al colesterol, el $6 \%$ presentaron cifras por encima del valor normal. Se observó hiperglicemia en $19 \%$ e hipoalbuminemia en el $88 \%$ de la población (Tabla 4).

La TGO aumentó en el $67 \%$ de los casos y la TGP en el 35\%. El $27 \%$ de los pacientes presentaron hipocalcemia, 37\% hiponatremia, 19\% hipocalemia y $19 \%$ hiperkalemia.

\section{Caracterización del perfil hematológico}

El $96 \%$ de los pacientes presentaron anemia, 15\% anemia microcítica y $8 \%$ anemia macrocítica; $75 \%$ presentaron linfocitopenia, 54\% leucopenia. El hematocrito descendió con respecto al valor inicial en el $96 \%$ de los pacientes durante la hospitalización y $15 \%$ presentaron trombocitopenia.

El $79 \%$ de los pacientes tenía recuento de linfocitos T CD4 $+<200 \mathrm{cel} / \mathrm{mm}^{3}$, con una media de 136 (Ds 199), carga viral con media de 228.209 copias (Ds 291.589), logaritmo de carga viral con media de 5 (Ds 1,26 ) (Tabla 4).

\section{Discusión}

Son pocos los estudios que describen las características epidemiológicas y clínicas de los pacientes hospitalizados con infección por VIH/SIDA en nuestro país. Hasta el momento no se han presentado estudios en los cuales se expongan suficientemente las alteraciones del perfil metabólico, endocrino y hematológico en estos pacientes hospitalizados. Las alteraciones presentadas en esta población inmunocomprometida con estadios avanzados de la enfermedad evidencian cambios que pueden deteriorar la calidad de vida y amenazar la misma ${ }^{6,7}$.

En varias series de estudios se ha reportado que la misma infección por el VIH, siendo una enfermedad crónica y sistémica, posiblemente provoca compromiso de múltiples órganos y del sistema endocrino $^{8-11}$.

Por otra parte, desde el inicio de la Terapia Antirretroviral combinada (ARTc), se han reportado varios efectos secundarios, entre ellos las alteraciones 
Perfil hormonal, metabólico y hematológico en adultos con el virus de inmunodeficiencia humana. Hospital Universitario de Neiva, Colombia

lipídicas, hematológicas, de la glucosa, óseas y diversos trastornos endocrinos (disfunción tiroidea, suprarrenal y gonadal), siendo estas alteraciones causadas por el compromiso directo de las glándulas por el propio virus, por infecciones oportunistas o neoplasias asociadas ${ }^{9,12}$.

Tabla 4. Perfil hormonal, metabólico y hematológico de los pacientes con infeccion por VIH hospitalizados en el HUHMP.

\begin{tabular}{|c|c|c|c|c|c|}
\hline \multicolumn{6}{|c|}{ Perfil hormonal } \\
\hline Variables & $\begin{array}{l}\text { \% Niveles } \\
\text { normales }\end{array}$ & $\begin{array}{c}\% \text { niveles } \\
\text { bajos }\end{array}$ & $\begin{array}{l}\% \text { Niveles } \\
\text { altos }\end{array}$ & Media /Ds & Valores de referencia \\
\hline Cortisol & & & & $85.9(94.2)$ & $4.3-20 \mathrm{Ug} / \mathrm{dl}$ \\
\hline Hombres $^{\mathrm{a}}{ }^{*} \mathrm{n}=29(\%)$ & $16(55.1)$ & $12(41.3)$ & $1(3.4)$ & & \\
\hline Mujeres ${ }^{\mathrm{b}} \mathrm{n}=16(\%)$ & $6(37.5)$ & $5(31.2)$ & $5(31.2)$ & & \\
\hline $\mathrm{TSH}(\mathrm{n}=52)$ & $32\left(61.5^{+}\right)$ & $3\left(5.7^{+}\right)$ & $17(32.6)$ & $4.1(5.5)$ & $0.4-4 \mathrm{uUI} / \mathrm{ml}$ \\
\hline T4 Libre $(\mathrm{n}=52)$ & $26(50.0)$ & $26(50.0)$ & $0(0.0)$ & $0.9(0.2)$ & $0.8-1.76 \mathrm{ng} / \mathrm{dl}$ \\
\hline FSH en hombres $(\mathrm{n}=34)$ & $32(94.1)$ & $2(5.8)$ & $0(0.0)$ & $6.4(6.9)$ & $1.7-12 \mathrm{mUI} / \mathrm{ml}$ \\
\hline LH en hombres $(n=34)$ & $27(79.4)$ & $1(2.9)$ & $6(17.6)$ & $4.5(2.9)$ & $1.1-7 \mathrm{mUI} / \mathrm{ml}$ \\
\hline Prolactina en hombres $(n=34)$ & $15(46.8)$ & $0(0.0)$ & $19(55.8)$ & $21.6(15.5)$ & $2.5-15 \mathrm{mg} / \mathrm{ml}$ \\
\hline Testosterona* & $0(0.0)$ & $10(100.0)$ & $0(0.0)$ & $5.6(3.7)$ & $3-10.6 \mathrm{ng} / \mathrm{ml}$ \\
\hline FSH en mujeres $(n=18)$ & $8(44.4)$ & $9(50.0)$ & $1(5.5)$ & $13.4(17.4)$ & $1.5-12 \mathrm{mUI} / \mathrm{ml}$; menopausia $17-95 \mathrm{mUI} / \mathrm{ml}$ \\
\hline LH en mujeres $(n=18)$ & $6(33.0)$ & $7(38.8)$ & $5(27.7)$ & $9.1(13.9)$ & $0.2-80 \mathrm{mUI} / \mathrm{ml}$; menopausia $8-33 \mathrm{mUI} / \mathrm{ml}$ \\
\hline Prolactina en mujeres $(n=18)$ & $10(55.5)$ & $0(0.0)$ & $8(44.4)$ & $21.6(15.5)$ & $2.5-20 \mathrm{ng} / \mathrm{ml}$ \\
\hline Estradiol** $(\mathrm{n}=8)$ & $4(50.0)$ & $4(50.0)$ & $0(0.0)$ & $39.8(37.1)$ & $\begin{array}{l}\text { Pre menopausia: } 18-575 \mathrm{pg} / \mathrm{ml} ; \text { Post } \\
\text { menopausia: } 0-58 \mathrm{pg} / \mathrm{ml}\end{array}$ \\
\hline \multicolumn{6}{|c|}{ Perfil metabólico - química sanguínea n = 52} \\
\hline Colesterol Total & $21(40.3)$ & $28(53.8)$ & $3(5.7)$ & $136.7(54.9)$ & $140-200 \mathrm{mg} / \mathrm{dl}$ \\
\hline HDL & $8(15.0)$ & $42(80.7)$ & $2(3.8)$ & $29.7(39.4)$ & $35-60 \mathrm{mg} / \mathrm{dl}$ \\
\hline LDL & $50(96.0)$ & $0(0.0)$ & $2(4.0)$ & $78.8(40.6)$ & $0-155 \mathrm{mg} / \mathrm{dl}$ \\
\hline VLDL & $29(55.7)$ & $0(0.0)$ & $23(44.2)$ & $33.2(26.0)$ & $2-30 \mathrm{mg} / \mathrm{dl}$ \\
\hline Triglicéridos & $28(53.8)$ & $1(1.9)$ & $23(44.0)$ & $171.1(131.8)$ & $35-150 \mathrm{mg} / \mathrm{dl}$ \\
\hline TGO & $14(26.9)$ & $3(5.7)$ & $35(67.3)$ & $61.1(47.4)$ & 15 - $37 \mathrm{UI} / \mathrm{L}$ \\
\hline TGP & $28(53.8)$ & $6(11.5)$ & $18(34.6)$ & $79.1(69.7)$ & $30-65 \mathrm{UI} / \mathrm{L}$ \\
\hline Creatinina & $37(71.1)$ & $11(21.1)$ & $4(7.6)$ & $0,79(0.3)$ & $0.6-1.3 \mathrm{mg} / \mathrm{dl}$ \\
\hline BUN & $38(72.0)$ & $7(13.4)$ & $7(13.4)$ & $13.4(10.8)$ & $7-18 \mathrm{mg} / \mathrm{dl}$ \\
\hline Albumina Total & $6(11.5)$ & $46(88.4)$ & $0(0.0)$ & $2.5(0.7)$ & $3.4-5 \mathrm{~g} / \mathrm{dl}$ \\
\hline Proteínas Totales & $26(50.0)$ & $14(26.9)$ & $12(23.0)$ & $7.2(1.3)$ & $6.4-8.1 \mathrm{~g} / \mathrm{dl}$ \\
\hline Glicemia Preprandial & $35(67.3)$ & $7(13.4)$ & $10(19.2)$ & $87.3(16.4)$ & $7^{\prime}-100 \mathrm{mg} / \mathrm{dl}$ \\
\hline \multicolumn{6}{|l|}{ Perfil hematológico $n=52$} \\
\hline Leucocitos & $19(36.5)$ & $28(53.8)$ & $5(9.6)$ & $5.6(4.7)$ & $4.8-10.810^{\wedge} 3 \times \mathrm{mm}^{\wedge} 3$ \\
\hline Linfocitos & $10(19.2)$ & $39(75.0)$ & $3(5.7)$ & $1.5(2.6)$ & $24-44 \%$ \\
\hline Hematocrito & $2(3.8)$ & $50(96.2$ & $0(0.0)$ & $33.2(5.2)$ & $42-52 \%$ \\
\hline Hemoglobina & $2(3.8)$ & $50(96.2)$ & $0(0.0)$ & $11(1.8)$ & $14-16 \mathrm{~g} / \mathrm{dl}$ \\
\hline VCM & $40(76.9)$ & $8(15.3)$ & $4(7.6)$ & $83.4(16.0)$ & $80-100 \mathrm{fl}$ \\
\hline Plaquetas & $40(76.9)$ & $8(15.3)$ & $4(7.6)$ & $271.7(128.7)$ & $150-45010^{\wedge} 3 \mathrm{x} \mathrm{mm}^{\wedge} 3$ \\
\hline \multicolumn{6}{|l|}{ Citometria y biología molecular } \\
\hline Recuento de CD4 & & $\mathrm{n}=43(\%)$ & & $136(199)$ & \\
\hline 1 & & $3(6.9)$ & & & $>500 \mathrm{cel} / \mathrm{mm} 3$ \\
\hline 2 & & $6(13.9)$ & & & $200-499 \mathrm{cel} / \mathrm{mm} 3$ \\
\hline 3 & & $34(79.0)$ & & & $<200 \mathrm{cel} / \mathrm{mm} 3$ \\
\hline Carga viral & & & & $228209(291589.2)$ & $<40$ copias $/ \mathrm{ml}$ \\
\hline Logaritmo de carga viral & & & & $5(1.26)$ & $<1.6 \log$ \\
\hline
\end{tabular}

(*) Sobre cada fila se relaciona la Frecuencia relativa de cada variable.

Ds=Desviación estándar

FSH: Folicle Stimulating Hormone; LH Luteinizing Hormone; TSH: Thyroid Stimulating Hormone, HDL: high-density lipoprotein, LDL: lowdensity lipoprotein; VLDL: very-low-density lipoprotein, TGO: Transaminasa Glutámico oxalacética, TGP: Transaminasa Glutámico pirúvica, BUN: Blood urea nitrogen, VCM: Volumen corpuscular medio.

*solo a 29 paciente masculinos se les reporte el resultado, ** solo a 16 paciente femeninas se les reporto los resultados, Los valores en paréntesis corresponden a porcentaje, ${ }^{* * *}$ Solo a 10 pacientes masculinos se les reporto resultados; ${ }^{* * * *}$ solo a 8 pacientes femeninas se les reporto resultados. HUHMP: Hospital Universitario Hernando Moncaleano Perdomo 


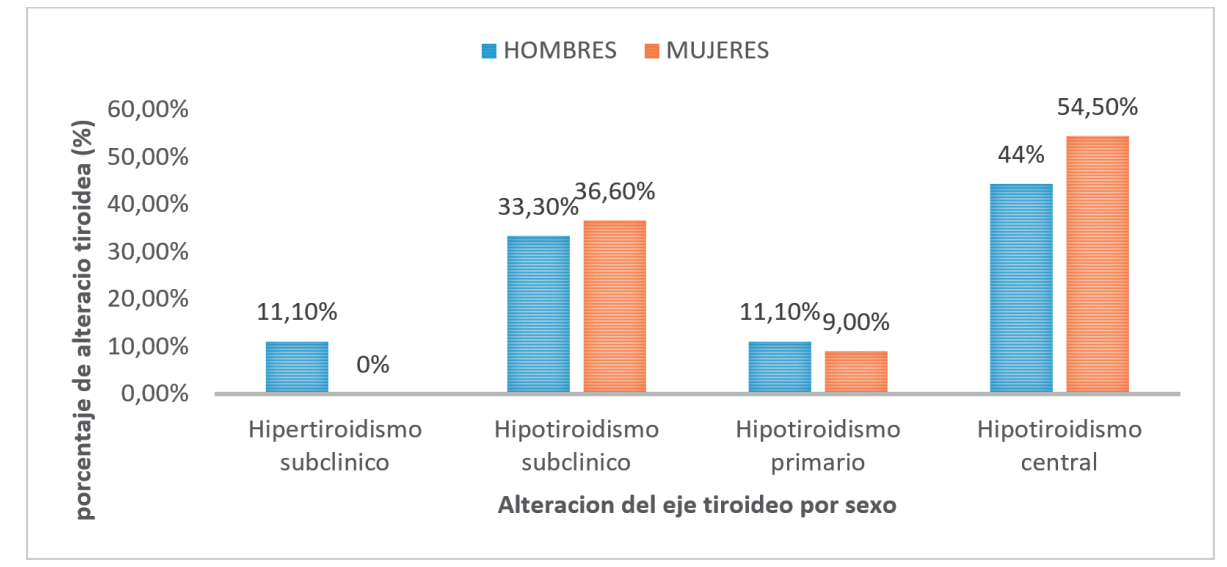

Figura 2. Representación de las alteración del eje tiroideo en los pacientes con infección por VIH hospitalizados en el HUHMP.

La dislipidemia es una de las complicaciones más frecuentes en los pacientes que reciben ARTc, con una prevalencia entre el 30 y $80 \%$, siendo la más frecuente la hipertrigliceridemia (40 a $80 \%$ ) seguida de hipercolesterolemia $(10 \text { a } 50 \%)^{9}$. Similar se encontró en nuestro estudio, la hipertrigliceridemia fue la alteración metabólica más frecuente (44\%).

La dislipidemia encontrada en nuestro estudio en un perfil francamente aterogénico, hipertrigliceridemia, con niveles de HDL bajos y elevación de los niveles VLDL; muy similar a lo observado en el estudio de Guerra M, et al. ${ }^{13}$; una cohorte de 30 pacientes con SIDA sin terapia antirretroviral previa en comparación con una cohorte sana; concluyeron que las alteraciones metabólicas de los lipídicos, están inducidas por el mismo proceso infeccioso oportunista agudo y por la progresión de la misma enfermedad, por lo cual es poco probable que los inhibidores de la proteasa que recibieron los pacientes (6/52) fuera la causa de hipertrigliceridemia reportada (44\%).

En el estudio de Mittal, et al. ${ }^{14}$ se evaluaron los factores de riesgo metabólicos después de iniciar un tratamiento antirretroviral, encontrando asociación entre el inicio de la terapia y el aumento significativo en los niveles de colesterol total y colesterol LDL. Valores diferentes fueron los encontrados en nuestra serie de estudio, con un colesterol total disminuido (54\%) y un LDL normal (96\%), dicho resultado puede ser explicado porque solo (12/52) 24\% recibieron HAART.

Rossibell Berenisse ${ }^{15}$, en su tesis de grado realizó un estudio con 132 pacientes VIH que se encuentran con un esquema de terapia con inhibidores de la proteasa (LPV-RPV) y su asociación con trastornos metabólicos y cardiovasculares; la alteración más frecuente fue hipertrigliceridemia (68/132) 51.51\% y HDL bajo 34 $\%$, en comparación con nuestro estudio fue del $44 \%$ y $80,7 \%$ respectivamente.

En estos pacientes la glucemia en ayunas generalmente es normal, pero tienen un riesgo de desarrollar diabetes de 4,6 veces mayor que en la población no $\mathrm{VIH}^{9}$. El $19 \%$ de nuestros pacientes presentó hiperglicemia y el $13 \%$ hipoglicemia asintomática.

En la disfunción tiroidea, no hay datos en pacientes hospitalizados en Colombia, siendo aun una controversia sobre la asociación entre terapia antirretroviral y el desarrollo de esta disfunción en estos pacientes; en nuestro país, Gómez, et $\mathrm{al}^{16}$ en su estudio de pacientes adultos ambulatorios reportaron que la mayor alteración del eje tiroideo fue el hipotiroidismo subclínico en pacientes que recibieron terapia antiretroviral, siendo mayor en los hombres; contrario a lo encontrado en nuestro estudio, donde la principal alteración fue el hipotiroidismo central.

Bongiovanni, et al. ${ }^{8}$ evaluaron la asociación entre hipotiroidismo subclínico y el inicio de la terapia antirretroviral, donde no encontraron relación estadística entre las variables, siendo igual la prevalencia en los grupos de comparación con y sin ARTc en un periodo de seguimiento de un año; sin embargo, encontraron correlación entre el aumento del colesterol total y los niveles de TSH, concluyendo que aquellos pacientes que desarrollaron hipercolesterolemia después del inicio de ARTc, son aquellos con mayor riesgo de hipotiroidismo subclínico ${ }^{8}$. La mayoría de estudios sugieren una mayor prevalencia de disfunción tiroidea en aquellos pacientes con VIH/SIDA desde el inicio de la era post HAART, sin ser clara dicha relación; siendo estavudina, didanosina y ritonavir los más relacionados 
con la presencia de hipotiroidismo subclínico ${ }^{8,9,12,17}$. Similar fue lo encontrado en nuestra serie de pacientes en la que todos se encontraban en estadio SIDA y con uso de inhibidores de proteasa (IP) solo en un $14 \%$.

En nuestra serie se encontró que el 12/29 hombres (41\%) y $5 / 16$ mujeres (31\%) participantes del estudio mostraron niveles bajos de cortisol, contrario a lo reportado en el estudio de Patterson, et al. ${ }^{18}$, una cohorte ambulatoria que no estaba recibiendo terapia antirretroviral y con recuento linfocitos TCD4 mayor a 250 celulas/l, en la cual los niveles de cortisol basales fueron significativamente más altos, describieron una relación entre el estrés, el sistema neuroendocrino, el sistema inmunológico y el riesgo de progresión a SIDA. Se ha descrito que a medida que los pacientes entran en un estado más inmunocomprometido, la probabilidad de insuficiencia de la glándula suprarrenal aumenta gradualmente, lo cual puede también ser causado por infecciones oportunistas.

El hipogonadismo es común entre los hombres infectados por el VIH, está en un 50\% de los hombres con SIDA $^{9}$ incluso entre los hombres que reciben terapia antirretroviral, pero con marcada disminución de la prevalencia. En nuestro estudio en el eje hipofisario gonadal, hay que aclarar que solo se lograron obtener reportes de niveles séricos de testosterona libre en 10/34 hombres. Encontrando hipogonadismo en dos pacientes, con recuento de linfocitos CD4 menor de 200 células $/ \mathrm{mm}^{3}$. Similar a lo encontrado en el estudio de Monroe, et al. ${ }^{19}$; que reportaron una prevalencia del hipogonadismo del $9.3 \%$, siendo un factor de riesgo aquellos pacientes con recuento de linfocitos CD4 menor de 200 células $/ \mathrm{mm}^{3}$.

La mayoría de las infecciones por VIH en las mujeres ocurren temprano en la vida reproductiva. En nuestro estudio se encontró que del total de las mujeres (18), 15 se encontraban en edad fértil y ocho tuvieron niveles de estradiol bajos, similar lo encontrado por Yalamanchi, et $\mathrm{al}^{20}$.

La hiperprolactinemia se documentó tanto en hombres como en mujeres, similar a lo reportado por Montero, et al. ${ }^{21,22}$; en dichos estudios se encontró que pacientes con VIH e infecciones oportunistas mostraron niveles más altos de prolactina. Estos hallazgos demostraron que la hiperprolactinemia no estaba relacionada con el tratamiento antirretroviral con inhibidores de proteasa, sino con la presencia de infecciones oportunistas activas en pacientes con SIDA.
Con respecto a los componentes de química sanguínea, se encontró hipoalbuminemia $(88,4 \%)$, hiponatremia (37\%) e hipocalemia (19\%); siendo similar lo observado en el estudio de Dao, et al. ${ }^{23}$, en la que constituyó un factor de riesgo para mortalidad en el primer año de inicio ARTc en aquellos pacientes con coinfección VIH - Tuberculosis.

Dentro de las alteraciones hematológicas, la anemia, leucopenia y trombocitopenia son hallazgos frecuentes durante la progresión de la infección por el VIH y en el SIDA, siendo la anemia la alteración más frecuente y con gran impacto en el deterioro de la calidad de vida, progresión de la enfermedad y mortalidad. Diversos estudios han demostrado que estas son multifactoriales, lo cual complica su diagnóstico diferencial y tratamiento adecuado, como se apreció en nuestro estudio ${ }^{24-28}$.

Dentro de los estudios realizados en Colombia, cabe destacar el de Laguado Vera, et al. ${ }^{28}$ de tipo observacional descriptivo, realizado en 54 pacientes. En él se reportó una prevalencia de anemia, leucopenia y trombocitopenia del $83.3 \%, 37 \%$ y $20.3 \%$, respectivamente. En comparación con nuestro estudio de 52 pacientes, se encontraron alteraciones de las mismas líneas celulares en el 96\%, 54\% y 15\% respectivamente. Los recuentos de linfocitos T-CD4 ${ }^{29}$ estuvieron por debajo de 200 células $/ \mathrm{mm}^{3}$, similar a lo reportado en el presente estudio.

Nuestro estudio aporta datos clínicos importantes en pacientes hospitalizados con infección VIH/SIDA, sin embargo, por el tipo de estudio y una población pequeña reclutada durante el periodo del estudio, no podemos inferir causa efecto sobre trastorno hormonal, endocrino-metabólico y hematológico asociados a la infección con VIH, si resulta del efecto directo del VIH o de las infecciones oportunistas o efecto directo de la HAART. Adicionalmente, los hallazgos encontrados en la literatura se limitan solo a los pacientes ambulatorios, dejando de lado el conocimiento en los pacientes hospitalizados.

Entre las limitaciones de nuestro estudio se encuentran: el excluir a pacientes hospitalizado con VIH que fallecieron, ya que ello pudo conllevar al desconocimiento del estado clínico y paraclínico de los pacientes más graves; el no contar con un grupo de control de pacientes hospitalizados sin VIH o SIDA o pacientes con VIH en categoría A o B, ya que toda la población sujeta a estudio estaba en categoría $\mathrm{C}$ con SIDA, al momento de diseñar el estudio no se tuvo 
disponibilidad para realizar las pruebas de vitamina D y somatomedina $\mathrm{C}$, por otro lado, algunas pruebas no fueron reportadas en una importante proporción de pacientes como fue el estradiol (8/52) y la testosterona $(10 / 52)$ en todos los pacientes estudiados, faltando más del $50 \%$ de los resultados.

\section{Conclusiones}

El perfil hormonal de los pacientes hospitalizados con VIH se caracteriza principalmente por afección del eje tiroideo y aumento de los niveles de prolactina. Los bajos niveles de cortisol en el grupo de estudio sugieren algún tipo de compromiso adrenal. Las características del perfil metabólico se relacionan con un perfil aterogénico. La hiponatremia es la alteración electrolítica de mayor importancia. Las alteraciones hematológicas involucraron las tres líneas celulares. Las principales infecciones oportunistas que motivaron la hospitalización fueron toxoplasmosis cerebral y tuberculosis.

Las múltiples afecciones encontradas en los pacientes hospitalizados pueden ser el resultado no solo del daño causado por el virus y el estado avanzado de enfermedad, sino también por las infecciones oportunistas y el compromiso multiorgánico, entre otros. Se requieren más estudios para evaluar el impacto de la detección temprana y el manejo intrahospitalario de dichas alteraciones en los pacientes con VIH.

\section{Conflicto de intereses}

Los autores declaran no tener conflictos de intereses ni fuentes externas de financiación del estudio.

\section{Consideraciones éticas}

El protocolo para la realización de esta investigación fue aprobado por el comité de ética e investigación del Hospital Universitario Hernando Moncaleano Perdomo de Neiva, Huila, Colombia, mediante el acta de aprobación 010-003 del 08-10-13. En toda la recolección de la información prevaleció el respeto a la dignidad de los sujetos de estudio y la protección de sus derechos y su privacidad. A los participantes se les informó el propósito y beneficios del estudio mediante el consentimiento informado para la utilización de la información clínica y de los exámenes de laboratorio, en coherencia con la Resolución 8430 de 1993 del Ministerio de Salud de Colombia y con aval del Comité de Bioética del HUHMP de Neiva, Huila, Colombia. Acta de aprobación 010-003 del 08-10-13.

\section{Referencias}

1. ONUSIDA/OMS. Situación de la epidemia del SIDA. 24 de noviembre de 2015.

2. Sistema Nacional de Vigilancia en Salud Pública (SIVIGILA). Boletín epidemiológico semanal número 52 de 2016.

3. Agudelo-Gonzalez S, Murcia-Sanchez F, Salinas D, Osorio J. Infecciones oportunistas en pacientes con VIH en el hospital universitario de Neiva, Colombia. 2007-2012. Infectio. 2015; 19(2): 52-59. doi: http:// dx.doi.org/10.1016/j.infect.2014.11.008.

4. Madge S, Smith CJ, Lampe FC, Thomas M, Johnson MA, Youle M, et al. No association between HIV disease and its treatment and thyroid function. HIV Med. 2007; 8(1): 22-27. doi: 10.1111/j.14681293.2007.00422.x.

5. Kasonde M, Niska RW, Rose C, Henderson FL, Segolodi TM, Turner K, et al. Bone mineral density changes among HIV-uninfected young adults in a randomised trial of Pre-Exposure prophylaxis with tenofovir-emtricitabine or placebo in Botswana. PLoS ONE. 2014; 9(3): e90111. doi: 10.1371/ journal.pone.0090111.

6. Granados C, Álvarez C, Prada G, Martínez F, Sarmiento C. Guía para el manejo de VIH/SIDA basada en la evidencia Colombia. 215; 1: 242.

7. Lamptey P, Johnson J, Khan M. El desafío mundial del VIH y el SIDA Washington, DC: Population Reference Bureau. 2006; 61(1): 28.

8. Bongiovanni M, Adorni F, Casana M, Tordato F, TincatiC, Cicconi P, etal. Subclinical hypothyroidism in HIV-infected subjects. J Antimicrob Chemother. 2006; 58(5): 1086-1089. doi: 10.1093/jac/dk1360.

9. Drnovsek ML, Portunato GB, San Martín PR, Virga MR, Losso M, Toibaro J, et al. Alteraciones endocrinometabólicas en pacientes ambulatorios con infección por Virus de la Inmunodeficiencia Humana. Rev Argent Endocrinol Metab. 2016; 53(4), 121-127. doi: 10.1016/j.raem.2016.09.002.

10. Chrousos GP, Zapanti ED. Hypothalamic-PituitaryAdrenal Axis in HIV Infection and Disease. Endocrinol Metab Clin North Am. 2014; 43(3): 791-806. doi: 10.1016/j.ecl.2014.06.002.

11. Rochira V, Guaraldi G. Hypogonadism in the HIV-infected man. Endocrinolo Metabol Clin North Am. 2014; 43(3): 709-730. doi: 10.1016/j. ecl.2014.06.005.

12. Abelleira E, De Cross GA, Pitoia F. Disfunción tiroidea en adultos infectados por el Virus de Inmunodeficiencia Humana. Med B Aires. 2014; 74(4): 315-320.

13. Guerra M, Alvarado M, Fonseca VL, Valbuena 
V. Comportamiento del metabolismo lipídico en pacientes con VIH/SIDA de Bogotá. Universitas Scientiarum, Rev Fac Ciencias. 2007; 12(2): 47-56.

14. Mittal A, Achappa B, Madi D, Chowta MN, Ramapuram JT, Rao S, et al. The development of metabolic risk factors after the initiation of the second line anti-retroviral therapy. J Clin Diagn Res. 2013; 7(2): 265-268. doi: 10.7860/ JCDR/2013/4740.2743.

15. Rossibell Berenisse OA. Tesis de grado: Alteraciones lipidicas en pacientes VIH/SIDA en terapia antiretroviral $(\mathrm{lpv} / \mathrm{rpv})$. Unidad técnica de infectología, Hospital Teodoro Maldonado Carbo. Guayaquiel Ecuador. 2014.

16. Gómez CH, Vesga JF, Lowenstein E, Suárez JO, Gil FA, Valderrama SL, et al. Detección de hipotiroidismo en un programa de atención de VIH/ SIDA en un hospital de Bogotá, Colombia. Rev Chil Infectol. 2011; 28: 59-63. doi: http://dx.doi. org/10.4067/S0716-10182011000100011.

17. Jain G, Devpura G, Gupta BS. Abnormalities in the thyroid function tests as surrogate marker of advancing HIV infection in infected adults. J Assoc Physicians India. 2009; 57: 508-510.

18. Patterson S, Moran P, Epel E, Sinclair E, Kemeny ME, Deeks SG, et al. Cortisol patterns are associated with T cell activation in HIV. PLoS ONE. 2013; 8(7): e63429. doi: 10.1371/journal.pone.0063429.

19. Monroe AK, Dobs AS, Palella FJ, Kingsley LA, Witt MD, Brown TT. Morning free and total testosterone in HIV-infected men: implications for the assessment of hypogonadism. AIDS Res Ther. 2014; 22; 11(1): 6. doi: 10.1186/1742-6405-11-6.

20. Yalamanchi S, Dobs A, Greenblatt RM. Gonadal function and reproductive health in women with Human Immunodeficiency Virus infection. Endocrinol Metab Clin North Am. 2014; 43(3): 731-741. doi: 10.1016/j.ecl.2014.05.002.

21. Montero A, Bottasso OA, Luraghi MR, Giovannoni AG, Sen L. Association between high serum prolactin levels and concomitant infections in HIV-infected patients. Hum Immunol. 2001; 62(2): 191-196.

22. Montero A, Bottasso OA, Luraghi MR, Giovannoni AG, Sen L. Galactorrhoea, hyperprolactinaemia, and protease inhibitors. Lancet. 2001; 357(9254): 473-474. doi: 10.1016/S0140-6736(05)71275-7.

23. Dao CN, Peters PJ, Kiarie JN, Zulu I, Muiruri P, Ong'ech J, et al. Hyponatremia, hypochloremia, and hypoalbuminemia predict an increased risk of mortality during the first year of antiretroviral therapy among HIV-infected Zambian and Kenyan women. AIDS Res Hum Retroviruses. 2011; 27(11):
1149-1155. doi: 10.1089/AID.2010.0345.

24. Denue BA, Gashau W, Bello HS, Kida IM, Bakki B, Ajayi B. Relation between some haematological abnormalities, degree of immunosuppression and viral load in treatment-naive HIV-infected patients. East Mediterr Health J. 2013; 19(4): 362-368.

25. Firnhaber C, Smeaton L, Saukila N, Flanigan T, Gangakhedkar R, Kumwenda J, et al. Comparisons of anemia, thrombocytopenia, and neutropenia at initiation of HIV antiretroviral therapy in Africa, Asia, and the Americas. Int J Infect Dis. 2010; 14(12): e1088-1092. doi: 10.1016/j.ijid.2010.08.002.

26. Wondimeneh Y, Muluye D, Ferede G. Prevalence and associated factors of thrombocytopenia among HAART naive HIV positive patients at Gondar university hospital, northwest Ethiopia. BMC Research Notes. 2014; 7: 5. doi: 10.1186/17560500-7-5.

27. Franzetti M, Adorni F, Oreni L, Van Den Bogaart L, Resnati C, Milazzo L, et al. Changes in the incidence of severe thrombocytopenia and its predisposing conditions in HIV-infected patients since the introduction of highly active antiretroviral therapy. J Acquir Immune Defic Syndr. 2014; 67(5): 493-498. doi: 10.1097/QAI.0000000000000347.

28. Laguado Vera LD, Arenas M, Rodríguez M, Gómez JC, Bonilla RA, Rojas G. Parámetros hematológicos y recuento de células T-CD4+ en pacientes VIH con síntomas digestivos en Bucaramanga, Colombia. Rev Cub Hematol Inmunol Hemoter. 2016; 32(4).

29. Álvarez M, Villa JP, Hidron A, Ocampo A, Restrepo L, Eusse A, et al. Perfil epidemiológico y clínico de pacientes colombianos hospitalizados con $\mathrm{VIH}$, enfermedades infecciosas del foro abierto. 2016; 3(suppl 1): 1531. doi: https://doi.org/10.1093/ ofid/ofw172.1233. 